\title{
The added value of semimicroelectrode recording in deep brain stimulation of the subthalamic nucleus for Parkinson disease
}

\author{
Pascal K. C. Jonker, B.Sc., ${ }^{1}$ J. Marc C. van DiJK, M.D., Ph.D., ${ }^{1}$ \\ Arjen L. J. van Hulzen, M.Sc., ${ }^{2}$ Teus van Laar, M.D., Ph.D., ${ }^{3}$ \\ Michiel J. Stahe, M.D., Ph.D., 1 and H. Louis Journée, M.D., Ph.D. ${ }^{1}$ \\ Departments of ${ }^{1}$ Neurosurgery, ${ }^{2}$ Radiology, and ${ }^{3}$ Neurology, University of Groningen, University Medical \\ Center Groningen, The Netherlands
}

\begin{abstract}
Object. Accurate placement of the leads is crucial in deep brain stimulation (DBS). To optimize the surgical positioning of the lead, a combination of anatomical targeting on MRI, electrophysiological mapping, and clinical testing is applied during the procedure. Electrophysiological mapping is usually done with microelectrode recording (MER), but the relatively undocumented semimicroelectrode recording (SMER) is a competing alternative. In this study the added value and safety of SMER for optimal lead insertion in the subthalamic nucleus (STN) in a consecutive cohort of patients with Parkinson disease (PD) was assessed.

Methods. Between 2001 and 2010, a consecutive single-center cohort of 46 patients with PD underwent DBS of the STN (85 lead insertions). After exclusion of 11 lead insertions for mostly technical reasons, 74 insertions were included for the assessment. Anatomical target localization was based on either 1.5-T MRI or fused 3-T MRI with $\mathrm{CT}$, with reference to anterior commissure-posterior commissure coordinates. Electrophysiological mapping was performed with SMER. Intraoperative clinical testing was dominant in determining the final lead position. The target error was defined as the absolute distance between the anatomical or electrophysiological target and the final lead position. The effect of SMER on anatomical target error reduction and final target selection was analyzed. Also, the anatomical and electrophysiological target error was judged against the different imaging strategies. For safety evaluation, the adverse events related to all lead insertions were assessed.

Results. The use of SMER significantly reduced the anatomical target error from 1.7 (SD 1.6) mm to 0.8 (SD 1.3) $\mathrm{mm}(\mathrm{p}<0.0001)$. In particular, the anatomical target error based on 1.5 -T MRI was significantly reduced by SMER, from 2.3 (SD 1.5) $\mathrm{mm}$ to 0.1 (SD 0.5) mm (p<0.001). Anatomical target error reduction based on 3-T MRI fused with CT was not significantly influenced by SMER $(\mathrm{p}=0.2)$, because the 3-T MRI-CT combination already significantly reduced the anatomical target error from 2.3 (SD 1.5) $\mathrm{mm}$ to 1.5 (SD 1.5) $\mathrm{mm}$ compared with 1.5-T MRI $(p=0.03)$. No symptomatic intracerebral hemorrhage was reported. Intracerebral infection was encountered in 1 patient following lead insertion.

Conclusions. Semimicroelectrode recording has added value in targeting the STN in DBS for patients with PD based on 1.5-T MRI. The use of SMER does not significantly reduce the anatomical target error in procedures with fused 3-T MRI-CT studies and therefore might be omitted. With the absence of hemorrhagic complications, SMERguided lead implantation should be considered a safe alternative to MER.

(http://thejns.org/doi/abs/10.3171/2013.8.FOCUS13289)
\end{abstract}

KEY WoRDS $\bullet \quad$ subthalamic nucleus $\bullet$ Parkinson disease
semimicroelectrode recording $\bullet$ deep brain stimulation

$\mathrm{S}$ INCE its breakthrough in 1987 , deep brain stimulation (DBS) has developed as an effective treatment for Parkinson disease (PD) ${ }^{3,4}$ Treatment with DBS leads to long-term improvements in motor function and

Abbreviations used in this paper: $\mathrm{DBS}=$ deep brain stimulation; $\mathrm{ICH}=$ intracerebral hemorrhage; $\mathrm{MER}=$ microelectrode recording; $\mathrm{PD}=$ Parkinson disease; $\mathrm{SMER}=$ semimicroelectrode recording; $\mathrm{STN}=$ subthalamic nucleus. benefits the quality of life. ${ }^{7,26}$ Several targets are available for stimulation, depending on the patient's symptomatology and age. ${ }^{7}$ Accurate lead placement is correlated with a better outcome. ${ }^{1}$ Therefore, to optimize the surgical positioning of the leads, a combination of anatomical targeting on MRI, electrophysiological mapping, and clinical testing is applied during the procedure. ${ }^{2}$

Intraoperative electrophysiological mapping corrects for image distortion, stereotactic frame inaccuracies, 
brain shift, ventricle passage, or pneumocephalus. ${ }^{11}$ Many centers successfully use microelectrode recording (MER) of single-cell activity for target refinement. ${ }^{10}$ However, MER comes with an increased risk of intracerebral hemorrhage (ICH) that correlates with the number of electrode passes, ${ }^{6,20}$ and MER is also reported to be time consuming and costly. ${ }^{19}$ Therefore, the Groningen University Medical Center uses the relatively undocumented semimicroelectrode recording (SMER) for intraoperative electrophysiological mapping of the subthalamic nucleus (STN). The SMER procedure differs from MER by the larger size and bluntness of the electrode tip. The neural activity of multiple cells is recorded instead of single-cell activity in MER. Nevertheless, SMER accurately distinguishes the increase in neural activity that demarcates the STN from adjacent structures. ${ }^{15,17,21,28}$ Therefore, SMER has the potential to improve the accuracy of lead placement without the disadvantages of MER.

The question remains to what extent intraoperative SMER contributes to optimal lead positioning within the STN. In this study, therefore, the added value and safety of SMER for optimal lead insertion in the STN in a consecutive cohort of patients with PD was assessed. In addition, the impact of improved imaging techniques on the relevancy of SMER was evaluated.

\section{Methods}

\section{Patient Population}

Between December 2001 and November 2010 a consecutive single-center cohort of 46 patients with advanced PD were subjected to SMER-guided DBS (85 lead insertions). Patient characteristics are shown in Table 1. All procedures were performed at the University Medical Center Groningen by the senior neurosurgeon (M.J.S.).

Patients who underwent operations based on a nonstandard imaging protocol (see below) were excluded for analysis of the target error. Also, lead insertions with insufficient SMER data and patients with a final lead position in a non-STN target were excluded. Therefore, 42 patients (74 lead insertions) were analyzed (Fig. 1).

For safety evaluation of the SMER procedure, the adverse events of all 85 lead insertions were assessed.

\section{Study Objectives}

The study objectives were as follows. 1) To define the target error of anatomical targeting and electrophysiological mapping with different imaging protocols, as compared with clinical targeting. 2) To define the influence of the imaging modality on the added value of SMER. 3) To assess the adverse effects of SMER.

\section{Technical Aspects of SMER}

The fundamental difference between MER and SMER is due to the size of the electrode. The electrode used in MER has a tip diameter of 5-10 $\mu \mathrm{m}$, with a typical impedance of $0.5-2 \mathrm{M} \Omega$. The semimicroelectrode has a blunt, cone-shaped tip with an end diameter of $75 \mu \mathrm{m}$ (Fig. 2). Consequently, the bigger tip has a 15- to 40 -fold larger contact surface and a lower impedance of 50-300 $\mathrm{k} \Omega$, which implies that the neural activity of multiple cells is recorded by SMER instead of single-cell activity by MER. The sharp pattern of action potentials measured by MER with typical amplitudes greater than $1 \mathrm{mV}$ is replaced in SMER by a relatively low-frequency spatial average of action potentials from a group of STN cells (also called multiunit recordings), with root mean square amplitudes of 0.1-0.5 $\mathrm{mV}$ (peak amplitudes may exceed 1 $\mathrm{mV})$. From the electrode dimensions, the theoretical sample volume of SMER is estimated to be approximately 2 magnitudes larger in comparison with MER.

\section{Imaging Protocols}

Between 2001 and 2004 intraoperative imaging was

TABLE 1: Demographic data in 46 patients with DBS for PD*

\begin{tabular}{|c|c|c|c|c|}
\hline Patient \& Electrode Data & Overall & After Exclusion & 1.5-T MRI & Fused 3-T MRI \& CT \\
\hline \multicolumn{5}{|l|}{ sex distribution (no.) } \\
\hline male & 30 & 26 & 9 & 17 \\
\hline female & 16 & 16 & 5 & 11 \\
\hline age in yrs at op $†$ & $59.6 \pm 9.5$ & $60.0 \pm 9.3$ & $61.1 \pm 9.2$ & $59.1 \pm 9.6$ \\
\hline \multicolumn{5}{|l|}{ no. of electrode implantation ops } \\
\hline bilat & 40 & 39 & 13 & 26 \\
\hline unilat & 6 & 3 & 1 & 2 \\
\hline \multicolumn{5}{|c|}{ hemisphere distribution (no. of insertions) } \\
\hline It & 46 & 39 & 14 & 25 \\
\hline $\mathrm{rt}$ & 39 & 35 & 11 & 24 \\
\hline \multicolumn{5}{|l|}{ periop imaging (no. of insertions) } \\
\hline 1.5-T MRI & 25 & 25 & 25 & NA \\
\hline fused 1.5-T MRI \& CT & 3 & 0 & NA & NA \\
\hline fused 3-T MRI \& CT & 57 & 49 & NA & 49 \\
\hline
\end{tabular}




\section{Semimicroelectrode recording in DBS for Parkinson disease}

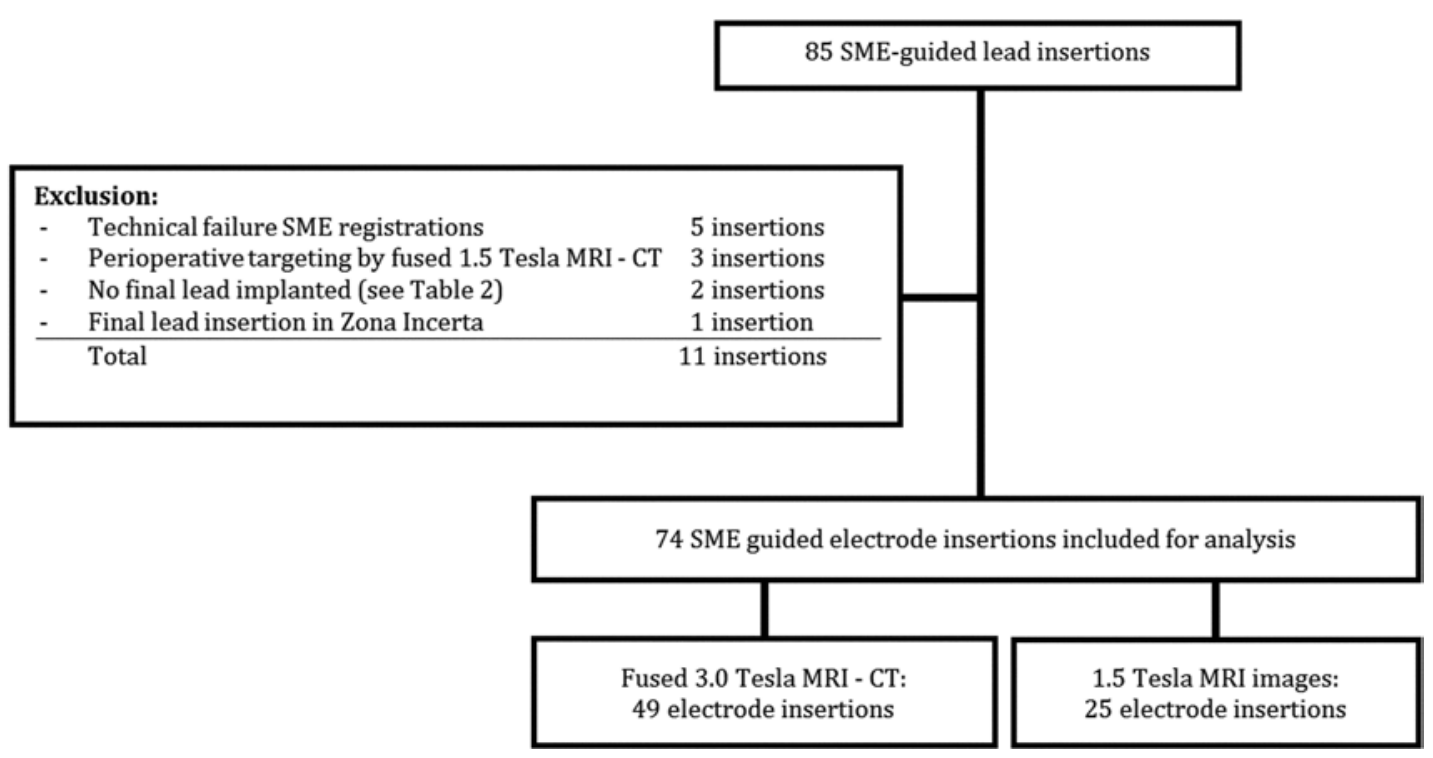

Fig. 1. Flowchart illustrating the inclusion of lead implantations for analysis of target error.

performed with a 1.5-T MRI magnet (SonataVision; Siemens). A 3D-volume T1, coronal T2, and a turbo inversion recovery series were fused using stereotactic planning software (@Target; Brainlab).

Since 2004, a 3-T MRI with a 32-element SENSE Head coil (Intera; Philips Medical Systems) has been used. The imaging protocol included a 3D-volume T1, and axial, coronal, and sagittal T2 scans. Also, axial and coronal T2 FLAIR and coronal short tau inversion recovery scans were made. The MRI sequences were made preoperatively and fused with an intraoperative axial (slice distance $1.5 \mathrm{~mm}$, thickness $2 \mathrm{~mm}$ ) CT scan (Sensation 64; Siemens) in a stereotactic planning system (iPlan 2.6; Brainlab).

Postoperative imaging was used to evaluate the final lead position. Between 2001 and 2004 postoperative MRI sequences and digitalized intraoperative skull radiographs were fused with anatomical target images in the @ Target software. Since 2004 postoperative CT imaging was fused with the target imaging in iPlan 2.6.

\section{Anatomical Targeting}

After rigid attachment of a Leksell G-Frame with a localizer box (Elekta Instruments) on the patient's head, intraoperative imaging was performed as described in the protocol. Localization of the STN was anatomically determined by the neurosurgeon based on the MRI data, both visually and in relation to anterior commissure-posterior commissure coordinates. The dorsolateral part of the STN was defined as the anatomical target.5,29 The same anatomical targeting protocol was used in both the 1.5-T and the 3-T MRI environment.

\section{Electrophysiological Mapping}

Using a custom-made manual micrometer and guiding needle, the semimicroelectrode (75 $\mu \mathrm{m}$; type IOFPH2274GV1 concentric Bipolar Clinical Electrode; Cormedica) was positioned $9 \mathrm{~mm}$ above the anatomical target and the baseline signal-to-noise ratio was assessed. Multistep recording was performed with 1-mm resolution to a maxi-

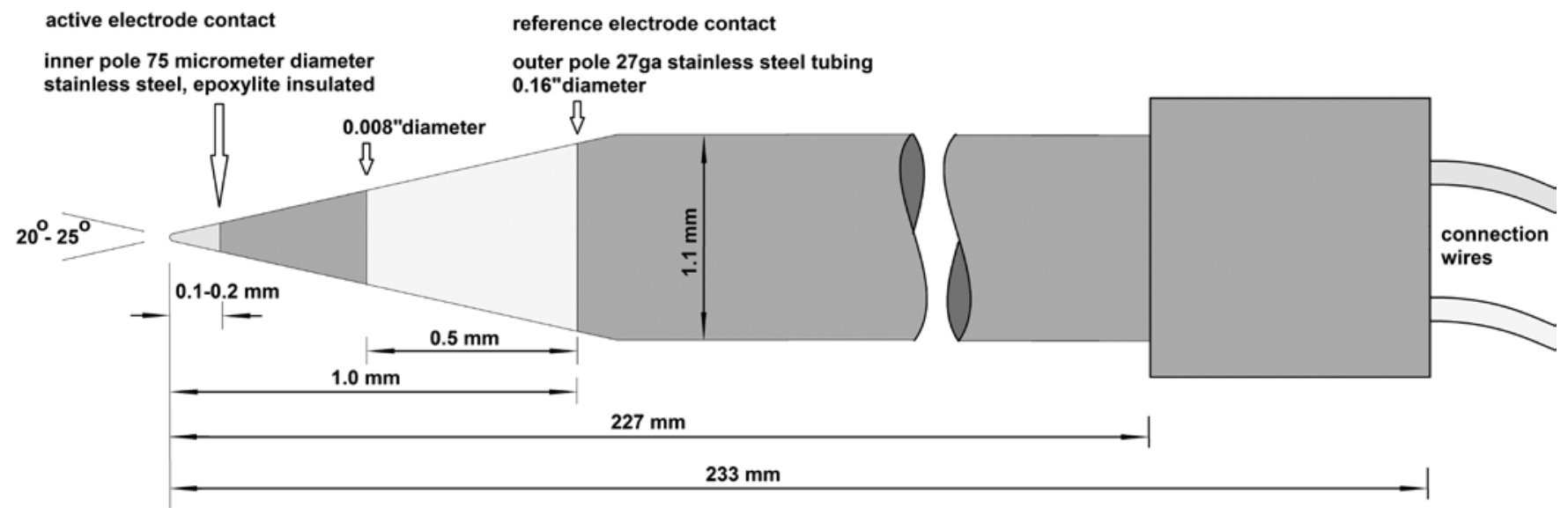

FIG. 2. Drawing of the semimicroelectrode with specific dimensions of the blunt tip. ga = gauge. 


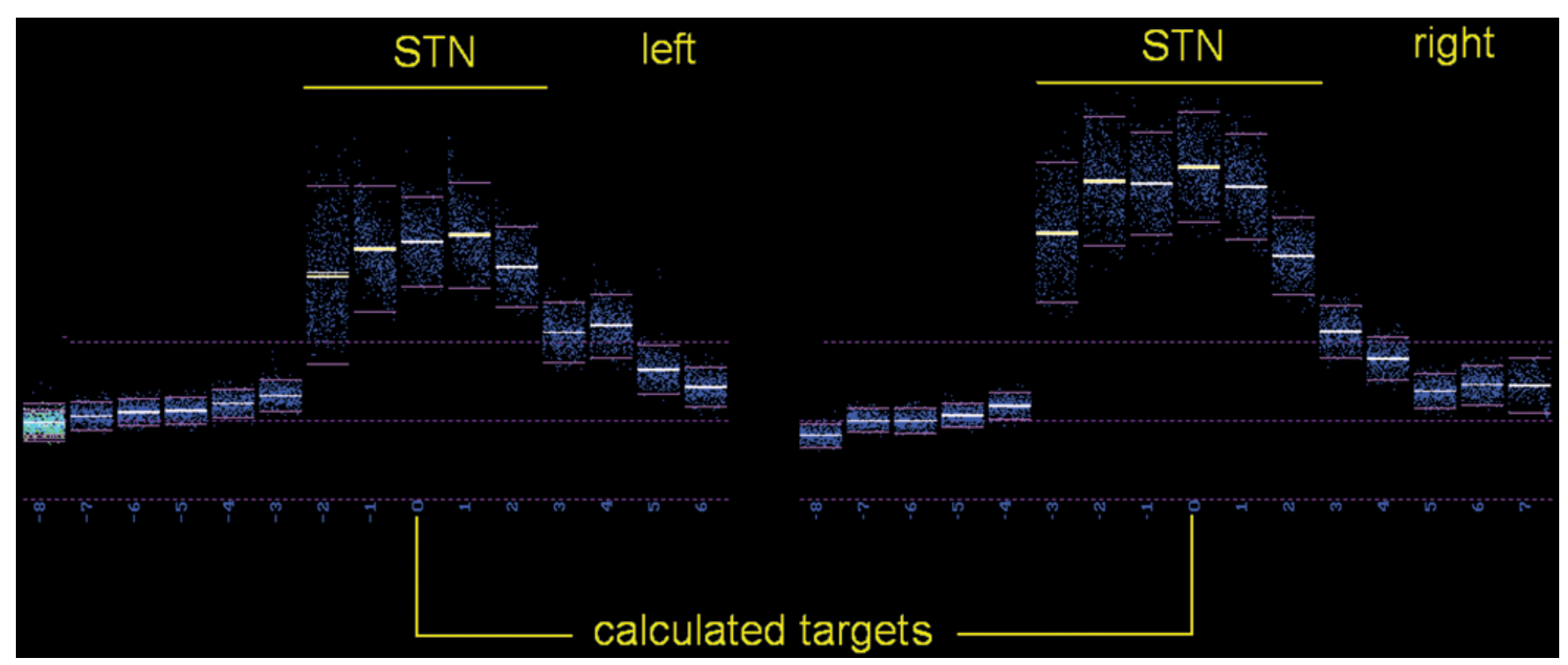

FIG. 3. Screenshot of an intraoperative SMER. The electrophysiological delineation of the bilateral STN is represented by the bars, with elevated levels of combined action potentials. The raw SMER data of a single measurement are visually expressed as a bar consisting of 500 dots. Each dot represents a root mean square amplitude over a timespan of $20 \mathrm{msec}$ (total measuring time 10 seconds).

mum of $8 \mathrm{~mm}$ below the anatomical target. The semimicroelectrode data were processed using software developed in-house, calculating a mean neural activity of 500 records during a measurement of 10 seconds (Fig. 3). The STN was defined by the area of significantly raised neural activity compared with adjacent recorded neural activity. The position with the highest recorded neural activity within the STN was indicated as the electrophysiological target.

\section{Intraoperative Clinical Testing}

The lead (Type 3389; Medtronic, Inc.) was inserted at the electrophysiological target coordinates through the same trajectory. Through this lead, low-frequency stimulation was increased step by step $(5 \mathrm{~Hz}$, maximum $7 \mathrm{~V}$ ) to verify a sufficient distance from the internal capsule. This was followed by high-frequency therapeutic pulse paradigms $(135 \mathrm{~Hz}$, maximum $4 \mathrm{~V})$ to evaluate the clinical effect. Clinical assessment of rigidity and bradykinesia was performed by a neurologist who specialized in movement disorders, together with intraoperative neurophysiological testing. ${ }^{12}$ The clinical target was defined as the position with the most optimal clinical effect during stimulation, which corresponded with the position of final lead placement in all insertions.

\section{Data Processing and Target Error}

Reconstruction of the surgical track was based on detailed procedural reports, and on intra- and postoperative imaging. Intracerebral tracks used for SMER, clinical evaluation, and final lead placement were reconstructed based on anatomical target position and on depth and angle information available from procedure reports.

The target error was defined as the absolute distance between the anatomical or electrophysiological target and the final lead position. The effect of SMER on reduction of the target error was assessed by comparing coordinates of anatomical, electrophysiological, and clinical targets. A subgroup analysis was performed on the insertions with 1.5-T MRI compared with fused 3-T MRI and CT studies.

\section{Statistical Analysis}

All statistical analysis was performed using Statistical Package for the Social Sciences version 16.0.1 (SPSS, Inc.). Patient demographics were calculated using descriptive statistics. Normality was assessed by performing the Shapiro-Wilk test. Data were assessed using the MannWhitney U-test and the Wilcoxon signed-ranks test to compare means between and within groups, respectively. A p value $<0.05$ was considered statistically significant.

\section{Results}

In $11(14.9 \%)$ of 74 insertions the anatomical, electrophysiological, and clinical target matched. In 4 insertions (5.4\%) the anatomical and electrophysiological target matched, but the clinical tests indicated a target adjustment. In 59 insertions (79.7\%) the anatomical target was adjusted based on SMER. In 33 of these 59 insertions the electrophysiological target corresponded with the clinical tests and no further adjustment occurred; in the other 26 insertions further adjustment of the electrophysiological target was done based on the clinical test results. The anatomical target error was 1.7 (SD 1.6) $\mathrm{mm}$. The use of SMER significantly reduced the anatomical target error, from 1.7 (SD 1.6) $\mathrm{mm}$ to 0.8 (SD 1.3) $\mathrm{mm}(\mathrm{Z}=-3.6$, $\mathrm{p}<$ $0.0001)$. Clinical testing contributed to a further significant improvement by reducing the electrophysiological target error to 0 (SD 0.0) $\mathrm{mm}(\mathrm{Z}=-4.8, \mathrm{p}<0.0001)$.

In 25 insertions the anatomical targeting was based 


\section{Semimicroelectrode recording in DBS for Parkinson disease}

on 1.5-T MRI. The anatomical, electrophysiological, and clinical target matched in $3(12 \%)$ of 25 insertions. In 2 insertions (8\%) the anatomical and electrophysiological target matched, but clinical test results indicated target adjustment. In 20 insertions (80\%) the anatomical target was corrected by SMER. The electrophysiologically refined target corresponded with clinical test results in all of these 20 insertions. The anatomical target error was 2.3 (SD 1.5) $\mathrm{mm}$. The use of SMER significantly reduced the anatomical target error from 2.3 (SD 1.5) $\mathrm{mm}$ to 0.1 (SD 0.5) $\mathrm{mm}(\mathrm{Z}=-3.9, \mathrm{p}<0.001)$. Further adjustment based on clinical test results was not significant $(Z=-1.3$, $\mathrm{p}=0.2$ ).

In 49 insertions the anatomical targeting was based on 3-T MRI fused with CT. In 8 insertions $(16.3 \%)$ the anatomical, electrophysiological, and clinical target matched. In 2 insertions (4.1\%) the anatomical and electrophysiological target matched, but clinical test results indicated target adjustment. In 39 insertions (79.6\%) the anatomical target was corrected by SMER. The electrophysiologically refined target corresponded with clinical test results in 13 insertions (26.5\%). In 26 insertions (53.1\%) the electrophysiologically refined target was further adjusted based on clinical test results. The anatomical target error in insertions based on fused 3-T MRI examinations was 1.5 (SD 1.5) $\mathrm{mm}$. The use of SMER reduced the anatomical target error from 1.5 (SD 1.5) $\mathrm{mm}$ to 1.1 (SD 1.4) $\mathrm{mm}(\mathrm{Z}=-1.3, \mathrm{p}=0.2)$. Clinical testing reduced the target error significantly, to $0(\mathrm{SD} 0.0) \mathrm{mm}(\mathrm{Z}$ $=-4.6, \mathrm{p}<0.0001)$.

Overall, the anatomical target error significantly declined when the imaging protocol changed from 1.5-T MRI to fused 3-T MRI-CT examinations $(\mathrm{Z}=-2.1, \mathrm{p}=$ $0.03)$, with a concomitant increase in electrophysiological target error $(\mathrm{Z}=-3.9, \mathrm{p}<0.0001)$.

None of the 85 insertions led to a symptomatic hemorrhagic complication. Postoperative imaging visualized a minimal amount of blood surrounding the tip of the final lead in 6 insertions. Two insertions involving 1 patient were aborted due to an intraoperative seizure during final lead placement. Intracerebral infection leading to a bipyramidal syndrome was reported following 1 insertion (Table 2).

\section{Discussion}

This study shows that SMER significantly reduced the overall anatomical target error. In particular, the anatomical target error based on 1.5-T MRI was significantly reduced by SMER, from 2.3 (SD 1.5) mm to 0.1 (SD 0.5) $\mathrm{mm}(\mathrm{p}<0.001)$. Anatomical target error reduction based on 3-T MRI fused with CT was not significantly influenced by SMER $(p=0.2)$, because the 3-T MRI-CT combination already significantly reduced the anatomical target error $(p=0.03)$. Furthermore, this study demonstrates that SMER is a safe procedure, because no symptomatic $\mathrm{ICH}$ was reported.

The overall reduction of the target error supports the hypothesis that SMER contributes significantly to intraoperative target selection. However, subgroup analysis showed that the quality of perioperative imaging influences the added value of the SMER and that intraoperative electrophysiological mapping is beneficial only in DBS procedures based on 1.5-T MRI examinations. Therefore, accurate lead insertion based on fused 3-T MRI-CT studies can be performed without electrophysiological mapping. The relatively small anatomical target error of 1.5 (SD 1.5) mm may not have clinical relevancy, and clinical testing can be used for further target refinement. This is in line with a recent study that reported good postoperative results without intraoperative electrophysiological mapping of the STN. ${ }^{8}$ Nevertheless, a prospective, randomized comparison between insertions with and without SMER is warranted for further substantiation of this hypothesis. Moreover, the demonstrated redundancy is only applicable to SMER in the 3-T MRI environment and clearly should not be extrapolated to the MER technique.

In the literature the application of SMER is scarcely reported. Hence, there are only limited data available directly comparing the accuracy of DBS procedures with and without neurophysiological mapping. The available studies on mapping during DBS mainly focus on postoperative clinical effect as primary outcome. ${ }^{10,21}$ Data about the intraoperative target error reduction and decision process leading to final target selection are inconsistently reported. Studies with SMER mainly describe adjustments in the orthogonal planes, whereas reports about MER use the average distance between targets..$^{10}$ Only 1 retrospective study directly compared the use of SMER and MER during a DBS procedure, reporting that the use of MER resulted in a better postoperative motor score, at the cost of a subtle but statistically significant deterioration of neuropsychological functions. ${ }^{23}$ Obviously, the lack of data in the literature further supports the need for consistent data collection and randomized comparison between SMER and MER, with accent on intraoperative target error reduction and clinical outcome.

A major disadvantage of DBS for symptomatic treat-

TABLE 2: Intraoperative and postoperative adverse events in 46 patients with DBS for PD

\begin{tabular}{|c|c|c|c|c|}
\hline Complication & $\begin{array}{l}\text { No. of Insertions } \\
\text { Involved }\end{array}$ & $\begin{array}{c}\text { Time Since } \\
\text { Electrode Insertion }\end{array}$ & Intervention(s) & Lingering Symptoms \\
\hline intraop seizure & 2 & intraop & procedure aborted & none \\
\hline minimal amount of blood (on postop imaging) & 6 & $0-7$ days & none & none \\
\hline superficial wound infection & 1 & 1 day & antibiotics & none \\
\hline intracerebral infection & 1 & 5 days & $\begin{array}{l}\text { antibiotics, removal } \\
\text { of DBS system }\end{array}$ & bipyramidal syndrome \\
\hline
\end{tabular}


ment of PD is the possibility of hemorrhagic complications. In that regard, electrophysiological mapping may cause an additional risk. A recent meta-analysis of STNDBS studies performed using mainly MER for electrophysiological mapping reported a symptomatic hemorrhage rate of $3.9 \% .^{13,25}$ In this study, no symptomatic ICH was reported. Other centers reported symptomatic hemorrhage rates of SMER varying between $0.0 \%$ and 3.2\%. ${ }^{14,16-18,22-24,27}$ An explanation for the reported difference in hemorrhagic complications between SMER and MER is the relatively blunt tip of the SMER. Another explanation may be the lower number of tracks needed during the insertion procedures, ${ }^{9}$ which also reduces procedural time and costs. ${ }^{19}$

\section{Conclusions}

Semimicroelectrode recording has added value in targeting the STN in DBS for patients with PD based on 1.5-T MRI. The use of SMER does not significantly reduce the anatomical target error in procedures with fused 3-T MRI-CT studies and therefore might be omitted. With the absence of hemorrhagic complications, SMERguided lead implantation should be considered a safe alternative to MER.

\section{Disclosure}

None of the authors report any relevant disclosures from the preceding 24 months.

Author contributions to the study and manuscript preparation include the following. Conception and design: van Dijk, Jonker, van Laar, Staal, Journee. Acquisition of data: Jonker, Journee. Analysis and interpretation of data: all authors. Drafting the article: van Dijk, Jonker. Critically revising the article: van Dijk, van Hulzen, van Laar, Staal, Journee. Reviewed submitted version of manuscript: all authors. Approved the final version of the manuscript on behalf of all authors: van Dijk. Statistical analysis: Jonker. Administrative/technical/material support: van Hulzen. Study supervision: Staal, Journee.

\section{References}

1. Anheim M, Batir A, Fraix V, Silem M, Chabardès S, Seigneuret E, et al: Improvement in Parkinson disease by subthalamic nucleus stimulation based on electrode placement: effects of reimplantation. Arch Neurol 65:612-616, 2008

2. Benabid AL, Chabardes S, Mitrofanis J, Pollak P: Deep brain stimulation of the subthalamic nucleus for the treatment of Parkinson's disease. Lancet Neurol 8:67-81, 2009

3. Benabid AL, Pollak P, Gross C, Hoffmann D, Benazzouz A, Gao DM, et al: Acute and long-term effects of subthalamic nucleus stimulation in Parkinson's disease. Stereotact Funct Neurosurg 62:76-84, 1994

4. Bergman H, Wichmann T, DeLong MR: Reversal of experimental parkinsonism by lesions of the subthalamic nucleus. Science 249:1436-1438, 1990

5. Coenen VA, Prescher A, Schmidt T, Picozzi P, Gielen FL: What is dorso-lateral in the subthalamic nucleus (STN)?-a topographic and anatomical consideration on the ambiguous description of today's primary target for deep brain stimulation (DBS) surgery. Acta Neurochir (Wien) 150:1163-1165, 2008

6. Deep-Brain Stimulation for Parkinson's Disease Study Group: Deep-brain stimulation of the subthalamic nucleus or the pars interna of the globus pallidus in Parkinson's disease. $\mathbf{N}$ Engl J Med 345:956-963, 2001
7. Fasano A, Daniele A, Albanese A: Treatment of motor and non-motor features of Parkinson's disease with deep brain stimulation. Lancet Neurol 11:429-442, 2012

8. Foltynie T, Zrinzo L, Martinez-Torres I, Tripoliti E, Petersen E, Holl E, et al: MRI-guided STN DBS in Parkinson's disease without microelectrode recording: efficacy and safety. J Neurol Neurosurg Psychiatry 82:358-363, 2011

9. Gorgulho A, De Salles AA, Frighetto L, Behnke E: Incidence of hemorrhage associated with electrophysiological studies performed using macroelectrodes and microelectrodes in functional neurosurgery. J Neurosurg 102:888-896, 2005

10. Gross RE, Krack P, Rodriguez-Oroz MC, Rezai AR, Benabid AL: Electrophysiological mapping for the implantation of deep brain stimulators for Parkinson's disease and tremor. Mov Disord 21 (Suppl 14):S259-S283, 2006

11. Halpern CH, Danish SF, Baltuch GH, Jaggi JL: Brain shift during deep brain stimulation surgery for Parkinson's disease. Stereotact Funct Neurosurg 86:37-43, 2008

12. Journee HL, Postma AA, Staal MJ: Intraoperative neurophysiological assessment of disabling symptoms in DBS surgery. Neurophysiol Clin 37:467-475, 2007

13. Kleiner-Fisman G, Herzog J, Fisman DN, Tamma F, Lyons KE, Pahwa R, et al: Subthalamic nucleus deep brain stimulation: summary and meta-analysis of outcomes. Mov Disord 21 (Suppl 14):S290-S304, 2006

14. Landi A, Parolin M, Piolti R, Antonini A, Grimaldi M, Crespi $\mathrm{M}$, et al: Deep brain stimulation for the treatment of Parkinson's disease: the experience of the Neurosurgical Department in Monza. Neurol Sci 24 (Suppl 1):S43-S44, 2003

15. Lanotte MM, Rizzone M, Bergamasco B, Faccani G, Melcarne A, Lopiano L: Deep brain stimulation of the subthalamic nucleus: anatomical, neurophysiological, and outcome correlations with the effects of stimulation. J Neurol Neurosurg Psychiatry 72:53-58, 2002

16. Lefaucheur JP, Gurruchaga JM, Pollin B, von Raison F, Mohsen N, Shin M, et al: Outcome of bilateral subthalamic nucleus stimulation in the treatment of Parkinson's disease: correlation with intra-operative multi-unit recordings but not with the type of anaesthesia. Eur Neurol 60:186-199, 2008

17. López-Flores G, Miguel-Morales J, Teijeiro-Amador J, Vitek J, Perez-Parra S, Fernández-Melo R, et al: Anatomic and neurophysiological methods for the targeting and lesioning of the subthalamic nucleus: Cuban experience and review. Neurosurgery 52:817-831, 2003

18. Lopiano L, Rizzone M, Bergamasco B, Tavella A, Torre E, Perozzo P, et al: Deep brain stimulation of the subthalamic nucleus: clinical effectiveness and safety. Neurology 56:552554,2001

19. McClelland S III: A cost analysis of intraoperative microelectrode recording during subthalamic stimulation for Parkinson's disease. Mov Disord 26:1422-1427, 2011

20. Palur RS, Berk C, Schulzer M, Honey CR: A metaanalysis comparing the results of pallidotomy performed using microelectrode recording or macroelectrode stimulation. J Neurosurg 96:1058-1062, 2002

21. Shin M, Lefaucheur JP, Penholate MF, Brugières P, Gurruchaga JM, Nguyen JP: Subthalamic nucleus stimulation in Parkinson's disease: postoperative CT-MRI fusion images confirm accuracy of electrode placement using intraoperative multi-unit recording. Neurophysiol Clin 37:457-466, 2007

22. Shin M, Penholate MF, Lefaucheur JP, Gurruchaga JM, Brugieres P, Nguyen JP: Assessing accuracy of the magnetic resonance imaging-computed tomography fusion images to evaluate the electrode positions in subthalamic nucleus after deep-brain stimulation. Neurosurgery 66:1193-1202, 2010

23. Temel Y, Wilbrink P, Duits A, Boon P, Tromp S, Ackermans L, et al: Single electrode and multiple electrode guided electrical stimulation of the subthalamic nucleus in advanced Parkinson's disease. Neurosurgery 61 (5 Suppl 2):346-357, 2007 


\section{Semimicroelectrode recording in DBS for Parkinson disease}

24. Vergani F, Landi A, Pirillo D, Cilia R, Antonini A, Sganzerla EP: Surgical, medical, and hardware adverse events in a series of 141 patients undergoing subthalamic deep brain stimulation for Parkinson disease. World Neurosurg 73:338-344, 2010

25. Videnovic A, Metman LV: Deep brain stimulation for Parkinson's disease: prevalence of adverse events and need for standardized reporting. Mov Disord 23:343-349, 2008

26. Weaver FM, Follett KA, Stern M, Luo P, Harris CL, Hur K, et al: Randomized trial of deep brain stimulation for Parkinson disease: thirty-six-month outcomes. Neurology 79:55-65, 2012

27. Yamada K, Goto S, Hamasaki T, Kuratsu JI: Effect of bilateral subthalamic nucleus stimulation on levodopa-unresponsive axial symptoms in Parkinson's disease. Acta Neurochir (Wien) 150:15-22, 2008

28. Yokoyama T, Sugiyama K, Nishizawa S, Tanaka T, Yokota N, Ohta S, et al: Neural activity of the subthalamic nucleus in Parkinson's disease patients. Acta Neurochir (Wien) 140:12871291, 1998

29. Yokoyama T, Sugiyama K, Nishizawa S, Yokota N, Ohta S, Akamine $\mathrm{S}$, et al: The optimal stimulation site for chronic stimulation of the subthalamic nucleus in Parkinson's disease. Stereotact Funct Neurosurg 77:61-67, 2001

Manuscript submitted July 10, 2013.

Accepted August 15, 2013.

Part of this work was presented in poster format during the First Intraoperative Neurophysiologic Monitoring Joint CrossAtlantic Educational Symposium at University Medical Center Groningen, The Netherlands, on November 5, 2010; as an abstract at the International Student Congress of (bio)Medical Science in Groningen, The Netherlands, on June 9, 2011; and at the 22nd Annual Congress of the American Society on Neurophysiological Monitoring in Orlando, Florida, on May 14, 2011.

Please include this information when citing this paper: DOI: 10.3171/2013.8.FOCUS13289.

Address correspondence to: J. Marc C. van Dijk, M.D., Ph.D., Department of Neurosurgery, AB71, University Medical Center Groningen, PO Box 30001, 9700 RB Groningen, The Netherlands. email: jmcvandijk@gmail.com. 